\title{
Sporadic hemiplegic migraine and CREST syndrome
}

\author{
Martin Pablo Grecco • Miguel Pieroni • \\ Marcela Otero · Jorge Luis Ferreiro • \\ María de Lourdes Figuerola
}

Received: 29 October 2009/Accepted: 6 January 2010/Published online: 4 February 2010

(C) Springer-Verlag 2010

\begin{abstract}
Hemiplegic migraines are characterised by attacks of migraine with aura accompanied by transient motor weakness. There are both familial and sporadic subtypes, which are now recognised as separate entities by the International Classification of Headache Disorders, edition II (ICHD-II). The sporadic subtype has been associated with other medical conditions, particularly rheumatological diseases. We report the case of a woman with sporadic hemiplegic migraine associated with CREST syndrome (calcinosis, Raynaud's phenomenon, esophageal dysmotility, sclerodactyly and telangiectasia). Since there is a close relationship between migraine and Raynaud's phenomenon, it could be speculated that the sporadic hemiplegic migraines in our patient might be secondary to CREST syndrome.
\end{abstract}

Keywords Migraine disorders · Hemiplegic migraine · Migraine with auras $\cdot$ CREST syndrome

\section{Introduction}

Hemiplegic migraines are characterised by fully reversible motor auras and have been recognised as a distinct group by the International Classification of Headache Disorders, edition II (ICHD-II) [1]. They present with two subtypes: genetic (familial hemiplegic migraine) and sporadic

M. P. Grecco $(\bowtie) \cdot$ M. Pieroni · M. Otero · J. L. Ferreiro Neurology, Hospital de Clínicas, Av. Córdoba 2351,

1120 Buenos Aires, Argentina

e-mail: martingrecco@yahoo.com

M. de L. Figuerola

Neurology, Hospital de Clínicas, CEDIE-CONICET,

Buenos Aires, Argentina (sporadic hemiplegic migraine) [1,2]. The latter has been associated with a number of medical conditions, such as tumours, vascular disorders and autoimmune diseases [3]. We report the case of a woman with sporadic hemiplegic migraine associated with CREST syndrome (calcinosis, Raynaud's phenomenon, esophageal dysmotility, sclerodactyly and telangiectasia).

\section{Case report}

A 34-year-old woman was referred to our clinic with a presumptive diagnosis of transient ischemic attack (TIA). She presented with episodes of transient hemiparesis that fully resolved without sequelae and were followed by severe headache. These episodes consisted of progressive left-sided weakness occurring in a stepwise pattern: first affecting the face, then the arm and finally the leg. Some of the episodes were preceded by sensory disturbances and, on other occasions, by scintillating scotomas. The symptomatology lasted no more than $30 \mathrm{~min}$ and was followed by an intense contralateral headache with a migrainous pattern lasting from 12 to $36 \mathrm{~h}$. The patient admitted having experienced similar episodes since adolescence, with a once-a-year frequency over the past 5 years. During the past year these attacks had become more frequent, increasing to one per month.

Her past medical history was remarkable for CREST syndrome, which first appeared at age 15 with Raynaud's phenomenon. She later developed skin induration in her hands and feet, calcinosis cutis and facial telangiectasias. These latter symptoms, which appeared approximately 15 years after Raynaud's phenomenon, led to the diagnosis of CREST syndrome. The presence of anti-centromere antibodies confirmed the diagnosis. She had no family 
history of migraine or any other neurological illnesses. Her usual medications included prednisone, nifedipine and aspirin. She was also taking levothyroxine for hypothyroidism. She had never taken oral contraceptives and was not on hormone replacement therapy.

The neurological examination was normal, and the laboratory panel showed a raised erythrocyte sedimentation rate of $48 \mathrm{~mm} / \mathrm{h}$ and a mild normocytic anaemia (haemoglobin $=11.5 \mathrm{mg} / \mathrm{dL}$ ). She had previously tested negative for anti-neutrophil cytoplasmic antibodies (ANCA), rheumatoid factor (RF), anti-DNA and anti-cardiolipin antibodies, hepatitis $\mathrm{B}$ and $\mathrm{C}$, HIV and syphilis. Magnetic resonance imaging (T1-weighted, T2-weighted and FLAIR sequences) showed only a few punctiform hyperintense white matter lesions on T2-weighted images. Diffusionweighted imaging (DWI) taken between 12 and $24 \mathrm{~h}$ after the hemiplegic attacks on two occasions were normal. Cerebral MRI angiography was also normal. Cerebrospinal fluid (CSF) analysis did not reveal any abnormalities. The electroencephalogram (EEG) showed no evidence of focal or general discharges. A transesophageal echocardiogram ruled out a patent foramen ovale (PFO). She was started on prophylactic treatment with topiramate $25 \mathrm{mg} / \mathrm{day}$, reaching a final dose of $75 \mathrm{mg}$ BID. After 9 months of followup, there was nearly a $50 \%$ reduction in both the frequency and severity of the migraine attacks with aura, and she did not experience further episodes of hemiplegic migraine.

\section{Discussion}

Hemiplegic migraines have been recognised as a separate entity by the ICHD-II (2004), comprising both familial (FHM) and sporadic (SHM) subtypes [1, 2]. The sporadic form is defined as "a migraine with aura including motor weakness but with no first or second degree relative with aura including motor weakness" [1]. SHM clinical characteristics are very similar to those of FHM, consisting of motor aura usually followed by other typical auras (mostly visual and sensory) and headache [2, 4, 5]. Motor deficits are usually unilateral, and most of them resolve in less than an hour. A large subset of patients also met the clinical criteria for basilar migraine during the attacks [1, 2, 4]. The main differential diagnoses include transient ischemic attacks (TIA), stroke and epilepsy [1, 2, 6]. Other entities with a similar clinical presentation, albeit uncommon, include MELAS (mitochondrial myopathy, encephalopathy, lactic acidosis and stroke-like episodes) and HaNDL (transient headache with neurological deficits and CSF lymphocytosis) [6]. The prognosis is generally good with complete recovery after the attacks. The frequency of the attacks tends to decrease with age, and the pattern becomes similar to migraine with aura. There are also reports in the literature describing cases of SHM attacks secondary to an underlying medical condition such as systemic lupus erythematosus (SLE), anti-phospholipid syndrome (APS), Sturge-Weber syndrome, hemifacial atrophy and tumours, among others [3]. This last group is also known as "symptomatic sporadic hemiplegic migraine" (symptomatic SHM) [3].

There are no uniform recommendations regarding treatment, although drugs such as propranolol, verapamil, flunarizine and naloxone have been used with variable degrees of response [2, 4]. It has been suggested that triptans and ergotamine derivatives are not useful and are even detrimental [2]. With regard to disease management, it seems reasonable to initiate preventive treatment only in those patients who have frequent and/or severe attacks. For the management of our patient, we started prophylactic treatment, taking into account both the frequency and intensity of her migraine attacks. She was started on topiramate given its favourable side effects profile in comparison with other treatments such as calcium channel blockers or naloxone in a young woman with a CREST syndrome as an associated pathology. She was also prescribed naproxen to relieve acute attacks.

To our knowledge this is the first case of symptomatic SHM associated with CREST syndrome. CREST syndrome is considered a subtype of limited scleroderma, consisting of calcinosis, Raynaud's phenomenon, esophageal dysmotility, sclerodactyly and telangiectasia [7]. The pattern of cutaneous involvement is limited to the face and distal extremities, sparing the trunk. Raynaud's phenomenon is usually the first symptom and is followed years later by skin thickening of the hands (sclerodactyly) as well as the other manifestations of the disease. Systemic involvement (e.g., lung fibrosis and kidney failure) tends to be less severe than in systemic sclerosis. Other associated conditions are pulmonary arterial hypertension, primary biliary cirrhosis and hypothyroidism. The presence of anti-centromere antibodies is a highly specific marker, though not very sensitive (50\%). In contrast to those with systemic sclerosis, the prognosis is generally better for patients with CREST [7].

Many reports in the literature associate Raynaud's syndrome with migraine [8, 9]. Compared to the general population, the prevalence of migraine is 2-4 times higher in patients with primary Raynaud's syndrome. In addition, Raynaud's syndrome appears to be more prevalent in patients with migraine [10]. There is also an increased prevalence of migraine in other diseases associated with Raynaud's, namely, SLE [11].

The involvement of small vessels is considered to be a relevant factor in the physiopathology of Raynaud's and CREST syndrome [7]. The mechanisms proposed to explain this involvement include an imbalance in 
vasomotor tone reactivity and changes in the architecture of the vessel walls, leading to obliterative fibrosis [7, 12]. These changes could sensitise the trigeminal vascular system, which is considered to play a major role in the physiopathology of migraine. Based on this we could speculate that the migraine attacks in our patient might be related to her CREST syndrome, although a casual association cannot be ruled out.

Acknowledgments The authors would like to thank Dr. Pablo A. López, Dr. Francisco Paulin and Dr. María Elina Grecco for their collaboration. Dr. Figuerola is Senior Researcher from CONICET.

Conflict of interest None.

\section{References}

1. Headache Classification Subcommittee of the International Headache Society (2004) The International Classification of Headache Disorders, 2nd edn. Cephalalgia 24 (Suppl 1):9-160

2. Thomsen LL, Ducros A (2005) Sporadic and familial hemiplegic migraines. In: Olesen J, Tfelt-Hansen P, Goadsby P (eds) The headaches, 3rd edn. Lippincott, Williams and Wilkins, Philadelphia, pp 583-585

3. Vetvik KR, Dahl M, Russell MB (2005) Symptomatic sporadic hemiplegic migraine. Cephalalgia 25:1093-1095

4. Thomsen LL, Olesen J (2004) Sporadic hemiplegic migraine. Cephalalgia 12:1016-1023

5. Thomsen LL, Eriksen M, Faerch S et al (2002) An epidemiological survey of hemiplegic migraine. Cephalalgia 5:361-375

6. Bhatia R, Desai S, Tripathi M et al (2008) Sporadic hemiplegic migraine: report of a case with clinical and radiological features. J Headache Pain 6:385-388

7. Varga, J (2008) Systemic sclerosis (scleroderma) and related disorders. In: Fauci A, Braunwald E, Kasper D et al (eds) Harrison's principles of internal medicine, 17th edn. McGraw-Hill, pp 2096-2106

8. Kaiser RS (1992) "Raynaud's disease" in migraineurs: one entity or two? Headache 32(9):463-465

9. Geraud G, Fabre N, Soulages X et al (1986) Migraine and the idiopathic Raynaud phenomenon. Rev Neurol (Paris) 142:638-640

10. Zahavi I, Chagnac A, Hering R et al (1984) Prevalence of Raynaud's phenomenon in patients with migraine. Arch Intern Med 144(4):742-744

11. Bernatsky S, Pineau CA, Lee JL, Clarke AE (2006) Headache, Raynaud's syndrome and receptor agonists in systemic lupus erythematosus. Lupus 12:671-674

12. Gabrielli A, Awedimento EV, Krieg T (2009) Scleroderma. N Engl J Med 360(19):1989-2003 